\title{
The role of polyamines in protein-dependent hypoxic tolerance of Drosophila
}

\author{
Paul Vigne ${ }^{1,2}$ and Christian Frelin*1,2
}

\author{
Address: ${ }^{1}$ Inserm, U615, Nice, F-06108, France and ${ }^{2}$ Univ Nice Sophia Antipolis, Nice, F-06108, France \\ Email: Paul Vigne - vigne@unice.fr; Christian Frelin* - cfrelin@unice.fr \\ * Corresponding author
}

Published: 2 December 2008

BMC Physiology 2008, 8:22 doi:10.1 186/1472-6793-8-22
Received: 8 September 2008

Accepted: 2 December 2008

This article is available from: http://www.biomedcentral.com/I472-6793/8/22

(C) 2008 Vigne and Frelin; licensee BioMed Central Ltd.

This is an Open Access article distributed under the terms of the Creative Commons Attribution License (http://creativecommons.org/licenses/by/2.0), which permits unrestricted use, distribution, and reproduction in any medium, provided the original work is properly cited.

\begin{abstract}
Background: Chronic hypoxia is a major component of ischemic diseases such as stroke or myocardial infarction. Drosophila is more tolerant to hypoxia than most mammalian species. It is considered as a useful model organism to identify new mechanisms of hypoxic tolerance. The hypoxic tolerance of flies has previously been reported to be enhanced by low protein diets. This study analyses the mechanisms involved.

Results: Feeding adult Drosophila on a yeast diet dramatically reduced their longevities under chronic hypoxic conditions $\left(5 \% \mathrm{O}_{2}\right)$. Mean and maximum longevities became close to the values observed for starving flies. The action of dietary yeast was mimicked by a whole casein hydrolysate and by anyone of the 20 natural amino acids that compose proteins. It was mimicked by amino acid intermediates of the urea cycle such as L-citrulline and L-ornithine, and by polyamines (putrescine, spermidine and spermine). $\alpha$-difluoromethylornithine, a specific inhibitor of ornithine decarboxylase, partially protected hypoxic flies from amino acid toxicity but not from polyamine toxicity. $\mathrm{N}^{\prime}$-guanyl-I,7 diaminoheptane, a specific inhibitor of elF5A hypusination, partially relieved the toxicities of both amino acids and polyamines.
\end{abstract}

Conclusion: Dietary amino acids reduced the longevity of chronically hypoxic flies fed on a sucrose diet. Pharmacological evidence suggests that the synthesis of polyamines and the hypusination of elF5A contributed to the life-shortening effect of dietary amino acids.

\section{Background}

Amino acid sensing is now recognized as an essential property of eukaryotic cells. It allows cells from developing organisms to adjust their rate of protein synthesis to the availability of amino acids. The availability/deprivation of amino acids is sensed by specific cellular protein kinases which target essential factors of the protein synthesis machinery. The availability of amino acids is sensed by the TOR pathway [1]. TOR activity is stimulated under nutrient rich conditions, hence leading to the phosphorylation of 4E-BP and an increase in the pool of free, active,
eIF4E. GCN2 kinase senses the opposite situation, i.e. the deficiency of a single amino acid species. GCN2 kinase is activated by uncharged tRNAs and it triggers a repression of global protein synthesis by phosphorylating the translation initiation factor eIF2 $\alpha$ [2].

Non growing tissues also sense available nutrients. For instance, it is well known that moderate dietary restrictions increase the longevity of a variety of organisms from yeast to mammals [3]. In Drosophila, the beneficial action of dietary restriction is largely reproduced by reducing die- 
tary proteins [4-6]. The mechanism involved is not known.

Chronic hypoxia is the situation in which tissues are exposed for long periods of time to oxygen tensions that are less than those required for optimum functioning. Chronic hypoxia is a major consequence of ischemic diseases such as stroke, myocardial infarction and venous thromboembolism and it clearly contributes to the progression of the diseases. The mechanisms by which chronic hypoxia induces hypoxic tissue damage are largely unknown and there is a need for innovative pharmacological strategies to enhance the hypoxic tolerance of ischemic tissues. Drosophila is increasingly used as a model system to analyze how whole organisms respond to exogenous stresses such as reduced oxygen [7]. We previously reported that chronically hypoxic flies retain normal activities but age at a faster rate than normoxic flies. We further observed that restriction of dietary proteins dramatically increases the longevity of hypoxic flies $[6,8]$. In this study we analyse the mechanisms involved in the diet dependent hypoxic tolerance. The results indicate that chronic hypoxic conditions unmask a life shortening effect of dietary amino acids which is mimicked by polyamines and partially prevented by inhibitors of polyamine synthesis. This study thus identifies a new amino acid sensing mechanism which determines the tolerance to chronic hypoxic stresses.

\section{Results}

\section{Dietary protein and amino acid induced hypoxic death}

Laboratory populations of Drosophila are usually raised on nutrient mixtures that consist of sucrose as a source of carbohydrates and heat inactivated yeast as a source of proteins and cofactors. We previously assessed the influences of sucrose and yeast on the longevity of flies maintained at atmospheric oxygen tension $\left(21 \% \mathrm{O}_{2}\right.$, normoxia) or under chronic hypoxic conditions $\left(5 \% \mathrm{O}_{2}\right)$ [6]. Results indicated that yeast and sucrose increased the longevity of normoxic flies. Sucrose increased the longevity of chronically hypoxic flies. Yeast did not. In addition dietary yeast suppressed the sucrose dependent longevity of chronically hypoxic flies. This paper deals with the mechanisms by which dietary yeast decreased the longevity of chronically hypoxic flies fed on a sucrose diet.

Figure 1A shows survivorship curves of hypoxic flies fed on a $10 \%$ sucrose diet supplemented with different concentrations of heat inactivated yeast. Dietary yeast decreased both the median and maximum longevities. Figure $1 \mathrm{~B}$ presents the dose response curve for the inhibitory action of yeast on the mean survival. It indicated a dose dependent and saturable action of yeast. Half maximum inhibition was observed at $0.16 \%$ yeast. Maximally effective concentrations of yeast decreased the mean sur- vival of hypoxic flies to about 2 days. A similar value (2.4 \pm 0.1 days, $\mathrm{n}=328$ ) was observed for starving flies that were exposed to the same hypoxic conditions.

Heat inactivated yeast is a complex mixture which comprises proteins, carbohydrates, nucleic acids, lipids and a variety of cell metabolites. We used a casein hydrolysate to evaluate the possible contribution of amino acids to the life shortening effect of yeast. Survivorship curves were established for hypoxic flies fed on a $10 \%$ sucrose diet supplemented with different concentrations of casein. Figure $1 \mathrm{C}$ shows that casein decreased both the median and maximum longevities of hypoxic flies. Figure 1D shows that the action of casein on the mean survival of hypoxic flies was dose dependent and saturable. Maximally effective doses of casein reduced the longevity of hypoxic flies to the same extent as maximally effective concentrations of yeast. The half maximum inhibition was observed at $0.01 \%$ casein. Thus, casein had the same maximum efficacy as yeast but it was 16 times more potent on a weight basis.

We then asked whether the toxicity of the casein hydrolysate was due to the whole amino acid mixture or to specific amino acid species. We exposed flies to a $10 \%$ sucrose diet supplemented with individual amino acids and determined survivorship curves under chronic hypoxic conditions. Amino acids were tested at the same concentration $(10 \mathrm{mM})$. All natural amino acids that compose proteins decreased the median and maximum longevities of hypoxic flies. The most active compounds were $\mathrm{L}$-asparagine and L-glutamine. They reduced the mean survival of the flies to $2.5-2.7$ days, close to the value observed for starving flies and flies fed on yeast rich or casein rich diets. The least active amino acid was L-lysine. It decreased the mean longevity to 6.1 days. Thus, a complete amino acid mixture was not required to reduce the longevity of hypoxic flies. Any one of the natural amino acids reproduced the action of casein. Figure $2 \mathrm{~A}$ ranks the different amino acids according to their actions on the mean longevity. This representation shows that no clear relationship could be defined between the life shortening effect of amino acids and their chemical structure.

We then defined the responses of hypoxic flies to different concentrations of L-proline, L-arginine and L-glutamine. The three amino acids reduced the median and maximum longevities of hypoxic flies in dose dependent manners (Figure 2B to 2D). Half maximum actions were observed at low $\mathrm{mM}$ concentrations. These potencies can be compared to that of casein. A $0.01 \%$ solution of casein hydrolysate, which produced a half maximum decrease in longevity, corresponded to a $1 \mathrm{mM}$ solution of amino acids (considering that the average molecular weight of an amino acid is $100 \mathrm{Da}$ ). Thus, $\mathrm{L}$-proline, $\mathrm{L}$-arginine and $\mathrm{L}$ - 
A

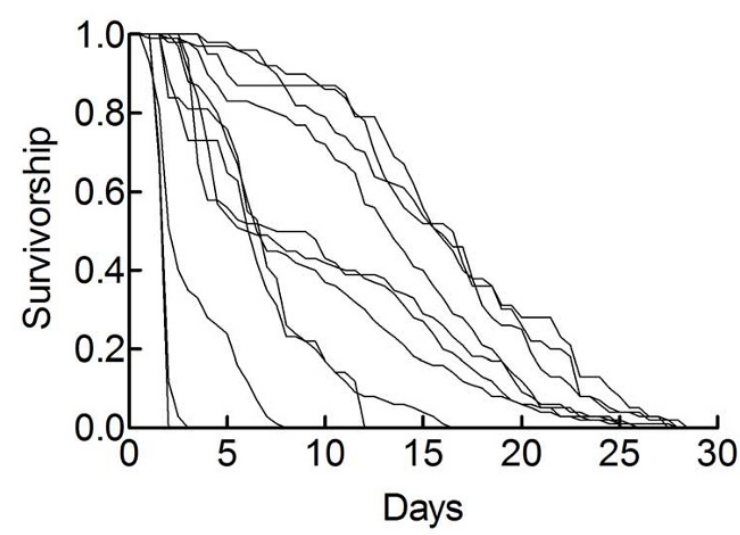

C

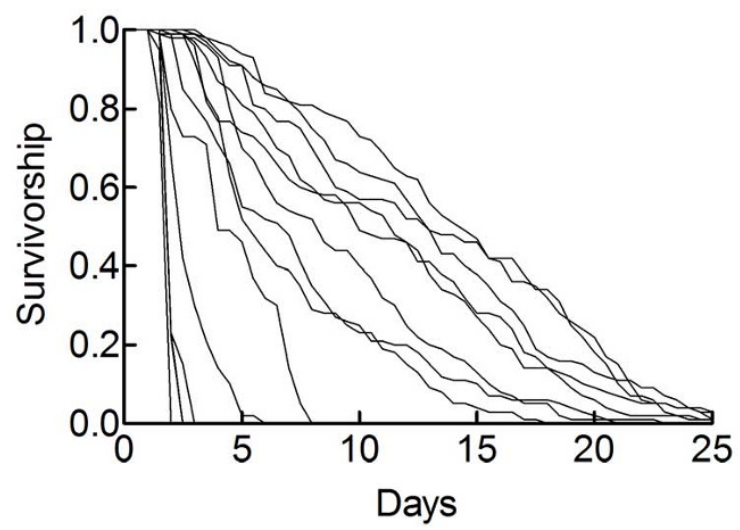

B

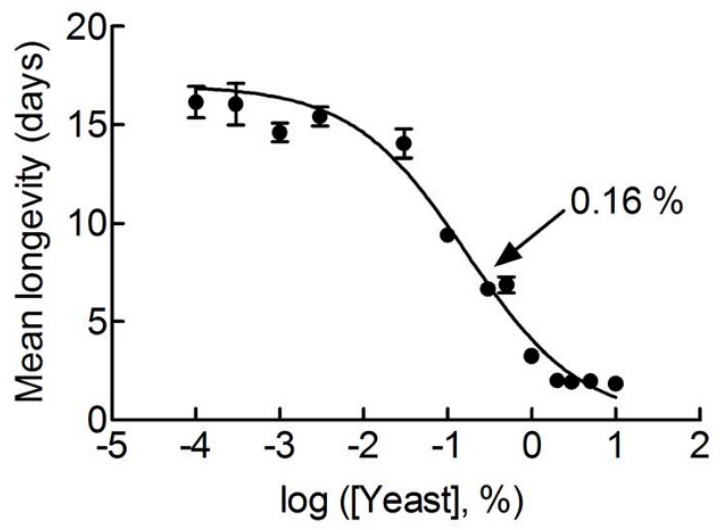

D

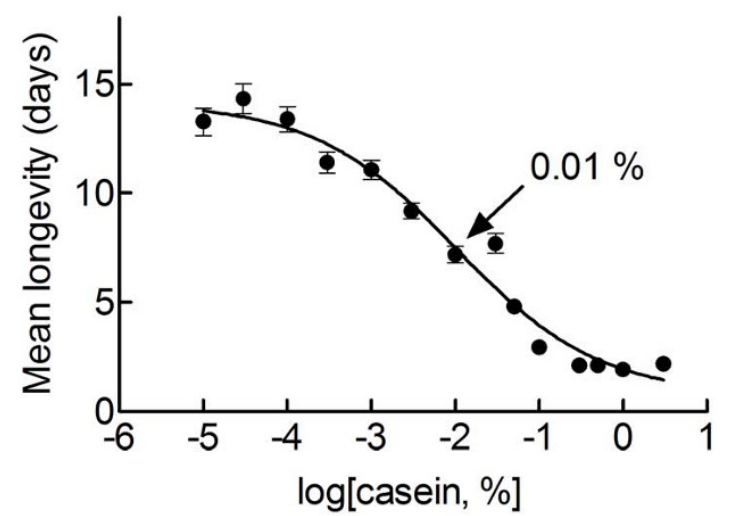

\section{Figure I}

Heat inactivated yeast and casein reduce the longevity of chronically hypoxic flies. Flies were exposed to a $10 \%$ sucrose diet supplemented with different concentrations of yeast (A, B) or of casein (C, D) and survivorship curves were determined under chronic hypoxia. A, C: Survivorship curves. Note that yeast and casein decreased both the median and maximum longevities. B, D. Dose response curves for the inhibitory action of yeast (B) and casein (D). Mean longevities \pm sem are indicated. The half maximum action of yeast was observed at a relative concentration of $0.16 \%$ (95\% confidence interval: $0.07-$ $0.38 \%$ ). The half maximum action of casein was observed at $0.01 \%$ casein $(95 \%$ confidence interval: $0.003-0.03 \%)$. The total numbers of flies under experimentation were 1722 and 1526 in experiments using yeast and casein respectively. Sample sizes used to define mean longevities were $40-323$.

glutamine were as potent as a casein hydrolysate. This indicated that amino acids quantitatively accounted for the action of casein. Yeast was 16 times less potent (Figure 1B). Different reasons may account for the lower potency of yeast. (i) Yeast provided less amino acid on a weight basis than the casein hydrolysate. Indeed yeast extracts are made up of only 50\% proteins. (ii) Free amino acids of the casein hydrolysate were more readily accessible to the flies than the undigested proteins provided by yeast powder. (iii) Some yeast component antagonized the toxicity of dietary proteins. For instance, odours from yeast have been shown to modulate the longevity extending effects of dietary restriction [9]. These results clearly caution about the use of poorly defined nutrient mixtures such as yeast extracts in longevity studies. The mechanism by which yeast decreased the longevity of hypoxic flies fed on a sucrose diet was not investigated further. We checked that, as expected, individual amino acids $(10 \mathrm{mM})$ were not toxic to normoxic flies. Clearly, their toxicities required hypoxic conditions to be observed. 

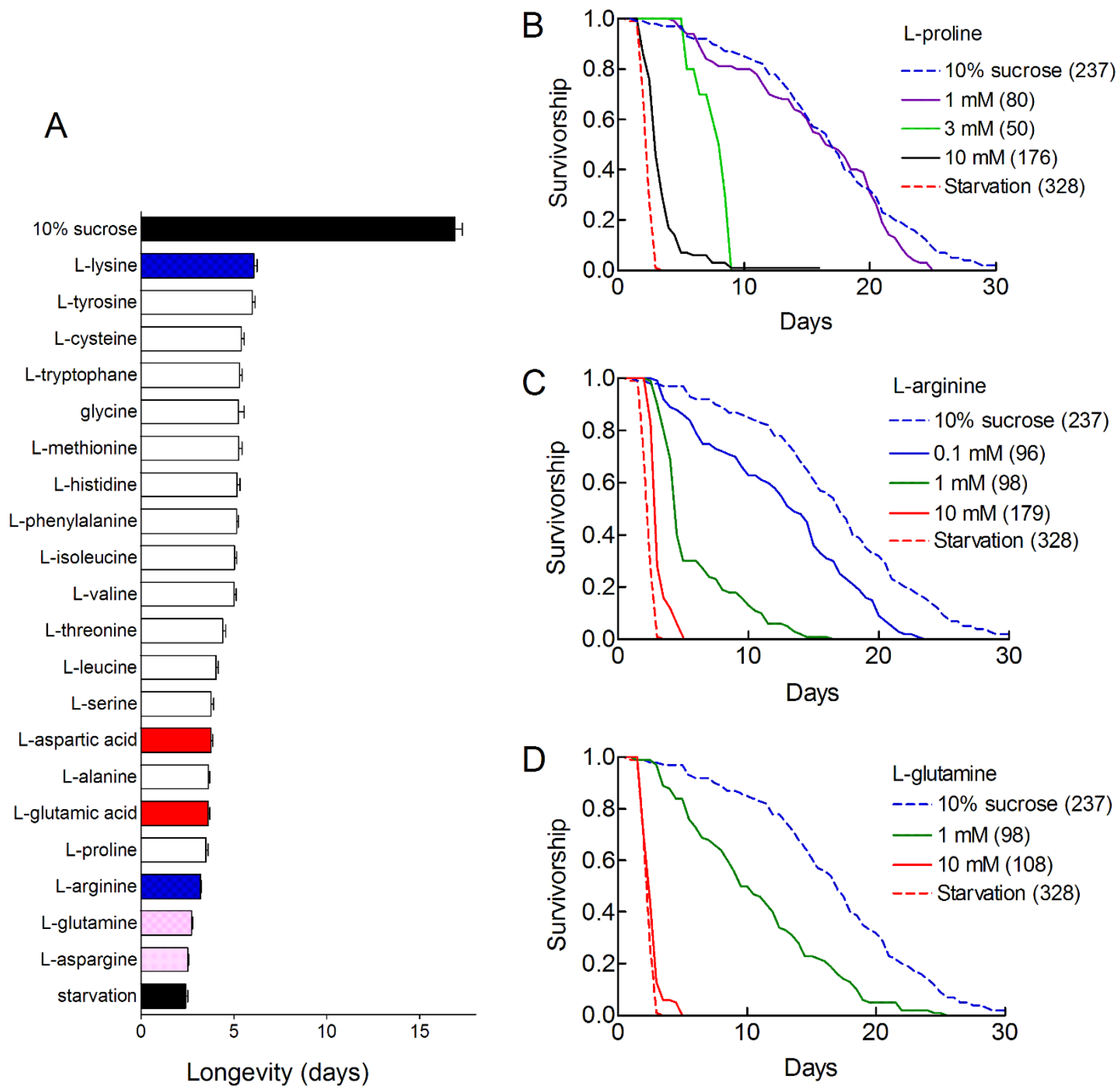

Figure 2

Natural amino acids reduce the longevity of chronically hypoxic flies. A. Flies were fed on a $10 \%$ sucrose diet supplemented with each individual amino acid $(10 \mathrm{mM})$ and survivorship curves were determined under chronic hypoxic conditions. Mean longevities \pm sem are shown. Amino acids are ranked according to their life-shortening effect. The top and bottom black bars show the two control situations which correspond to a pure sucrose diet and starvation conditions. All differences with the pure sucrose condition were statistically significant $(p<0.000 \mathrm{I}$, log rank test). Colour codes were used to label basic amino acids (blue), acidic amino acids (magenta) and amidinated amino acids (red). The total number of flies used was 234l. Sample sizes were 70-237. B-D. Dose dependent actions of L-proline, L-arginine and L-glutamine. Hypoxic flies were fed on a $10 \%$ sucrose medium supplemented with the indicated concentrations of amino acids and survivorship curves were determined. The dotted lines show the survivorship curves obtained for hypoxic flies fed on a pure sucrose diet (blue) or under starvation conditions (red). Sample sizes are indicated in parentheses. Note that low $\mathrm{mM}$ concentrations of amino acids produced near half maximum reductions in the median and maximum longevities of the flies. 


\section{L-citrulline, L-ornithine and polyamines increased hypoxic death}

The absence of clear structure activity relationship (Figure $2 \mathrm{~A}$ ) suggested that amino acids might act via a very general metabolic pathway. The urea cycle is a likely candidate for the most active amino acids are closely linked to the urea cycle. Arginine is an intermediate of the cycle. Aspartic acid is a substrate for argininosuccinate synthase. It is produced from oxalacetate using transaminating reactions (Figure 3). The urea cycle comprises two amino acid intermediates which are not incorporated into proteins: Lornithine and L-citrulline. We therefore asked whether Lcitrulline and L-ornithine also reduced the longevity of hypoxic flies. Survivorship curves were established for hypoxic flies fed on a $10 \%$ sucrose diet supplemented with $10 \mathrm{mM}$ L-citrulline or L-ornithine. Figure 4 shows that the two amino acids decreased the median and maximum longevities of hypoxic flies to the same extent as starvation conditions. Their actions were dose dependent.
Half maximum actions were observed at low mM concentrations as observed for natural amino acids (Figure 2). Thus, dietary L-ornithine and L-citrulline reproduced all actions of individual amino acids and casein.

\section{Pharmacological evidence for a role of polyamines in hypoxic tolerance}

One well known function of L-ornithine is to feed the polyamine synthetic pathway (Figure 3). We therefore asked whether polyamines reduced the longevity of hypoxic flies. Figure $4 \mathrm{~A}$ shows that putrescine, spermine and spermidine decreased the mean longevities of hypoxic flies fed on a pure sucrose diet. Mean longevities were 4-8 days, similar to the values observed in the presence of the least active amino acids. Putrescine, spermine and spermidine were well tolerated by normoxic flies. They did not induce an excess mortality in the normoxic toxicity assay.
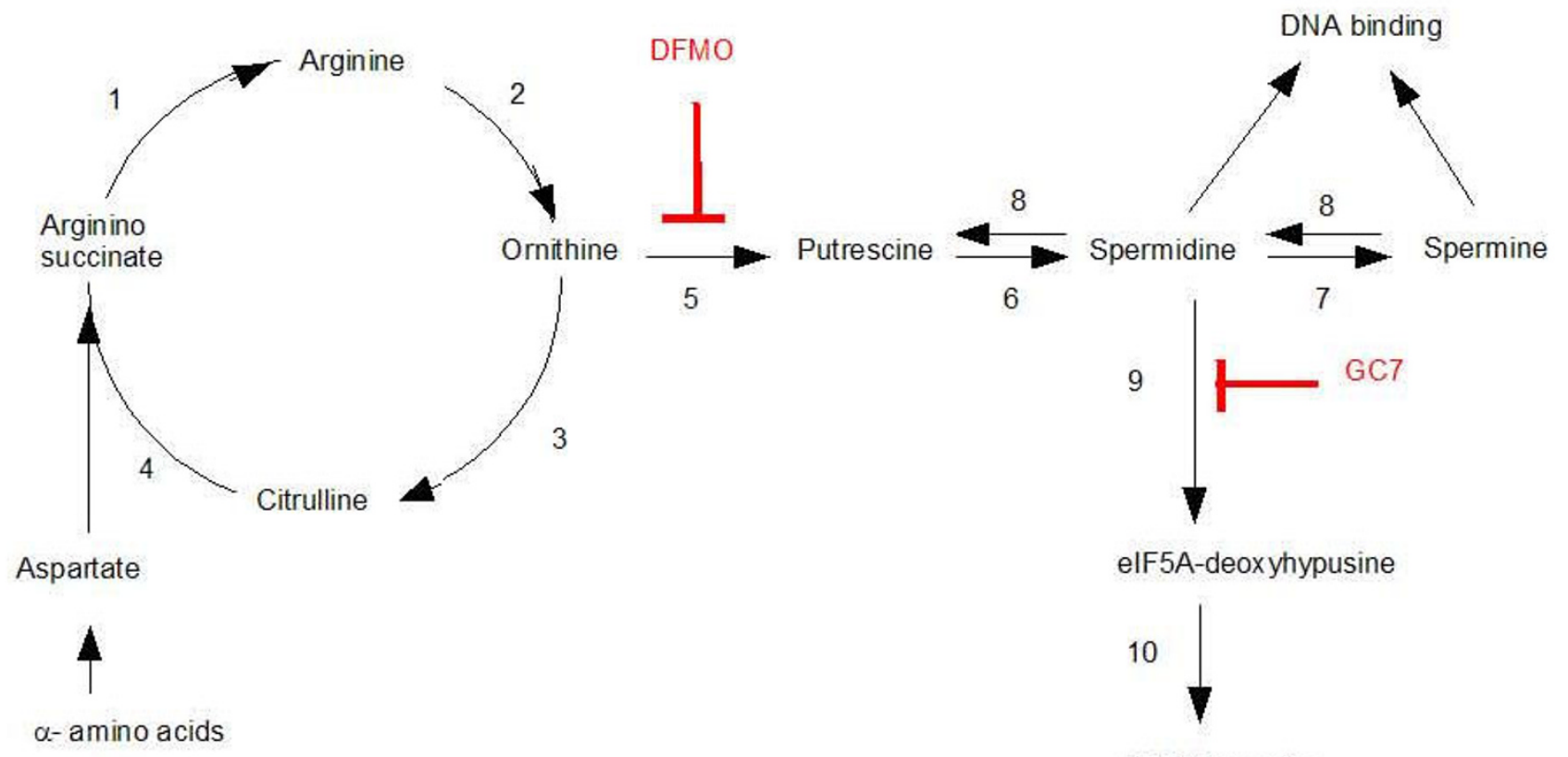

elF5A-hypusine

\section{Figure 3}

The synthesis of polyamines and the hypusination of elF5A. Enzymes are: arginosuccinate lyase (I), arginase (2), ornithine carbamoyl transferase (3), arginosuccinate synthase (4), ornithine decarboxylase (ODC) (5), spermidine synthase (6), spermine synthase (7). Spermine synthase and spermidine synthase also require decarboxylated S-adenosylmethionine which is produced by S-adenosylmethionine decarboxylase. Polyamines catabolism (8) is achieved by spermine/spermidine $\mathrm{N}^{\prime}$-acetyltransferase and a peroxisomal flavoprotein polyamine oxidase. The formation of hypusine ( $\mathrm{N}^{\varepsilon}$-(4-amino-2-hydroxybutyl)lysine) occurs in two steps. Deoxyhypusine synthase (9) transfers the 4-aminobutyl moiety of spermidine to the $\varepsilon$-amino group of a specific lysine residue of elF5A. Deoxyhypusine hydroxylase (I0) hydroxylates elF5A-deoxyhypusine. In yeast and mammals, only hypusinated forms of elF5A are active. DFMO inhibits ODC, the rate limiting enzyme for polyamine synthesis. GC7 inhibits deoxyhypusine synthase (DHS). The polyamines synthetic enzymes, ODC and S-adenosylmethionine decarboxylase are up regulated in response to a decrease in polyamines. Spermine/spermidine $\mathrm{N}^{\prime}$-acetyltransferase which mediates the retroconversion of polyamines is up regulated by spermine and spermidine. 
A

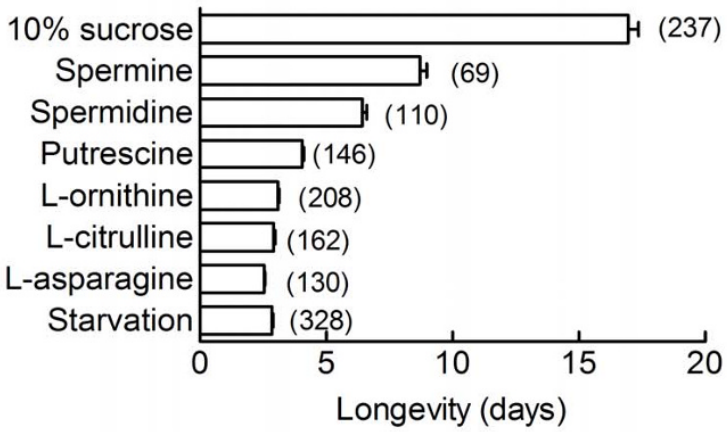

B

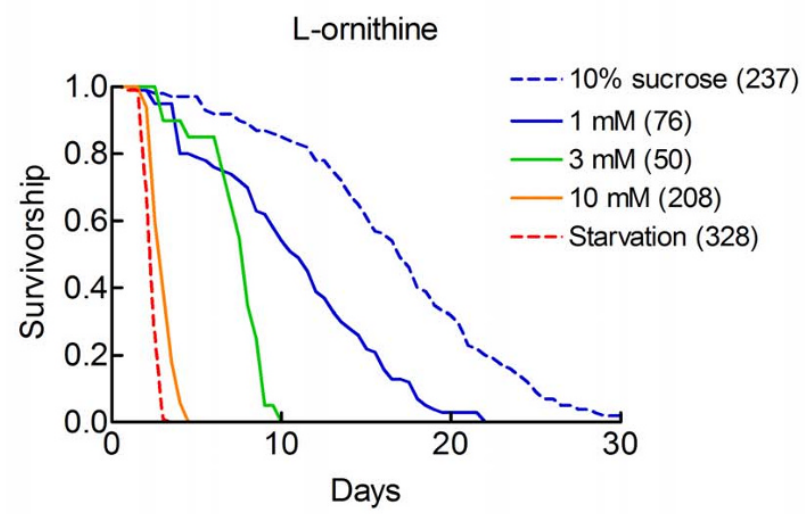

C

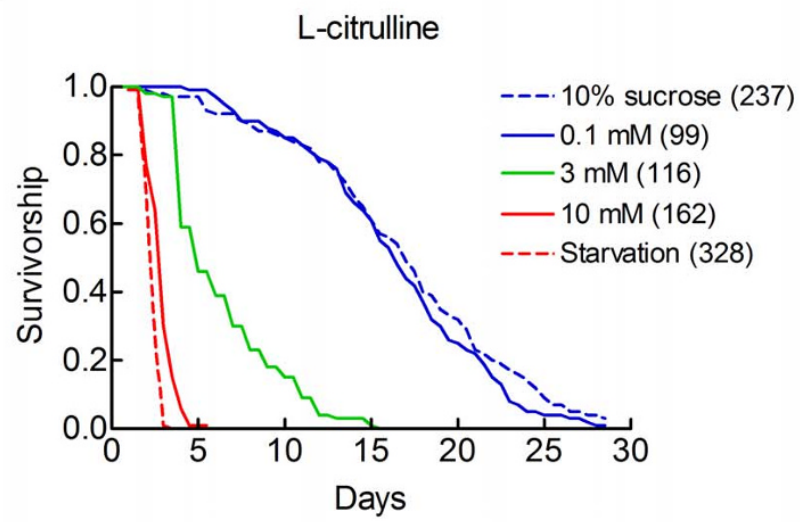

\section{Figure 4}

Life-shortening effects of L-ornithine, L-citrulline and polyamines. A. Hypoxic flies were fed on a $10 \%$ sucrose medium supplemented with $10 \mathrm{mM}$ of the indicated compounds and survivorship curves were determined. Mean longevities \pm sem are shown. Sample sizes are indicated in parentheses. B, C: Dose dependent actions of L-ornithine (B) or of L-citrulline (C) on the survival of hypoxic flies. Hypoxic flies were fed on a $10 \%$ sucrose diet supplemented with the indicated concentrations of amino acids and survivorship curves were determined. The dotted lines show the survivorship curves obtained for flies fed on pure sucrose diet (blue) or under starvation conditions (red). Sample sizes are indicated in parentheses. Note that low $\mathrm{mM}$ concentrations of L-ornithine and L-citrulline produced near half maximum reductions in the median and maximum longevities of the flies. Survivorship curves of flies fed on $10 \mathrm{mM}$ putrescine or $10 \mathrm{mM}$ spermidine are presented in Figure 5 (E and F). 
Ornithine decarboxylase (ODC) is the rate limiting enzyme for polyamine synthesis in mammals. DFMO $(\alpha-$ difluoromethylornithine) is a specific inhibitor of ODC in mammals [10] and insects [11]. Figure 5 shows that DFMO increased the median and maximum longevities of hypoxic flies fed on casein, L-asparagine or L-ornithine. Percentage changes in the mean longevity by DFMO were 29 to $86 \%$. We observed however that flies exposed to DFMO were shorter lived than flies fed on a pure sucrose diet (14.2 days). Thus, DFMO partially reversed the lifeshortening effect of casein and amino acids.

Figure $5 \mathrm{~A}$ shows that DFMO did not modify the longevity of starving hypoxic flies or of hypoxic flies fed on a sucrose diet. Thus, DFMO acted specifically on hypoxic flies that were fed on amino acids.

Figure $5 \mathrm{E}$ and $5 \mathrm{~F}$ further shows that DFMO did not increase the longevity of hypoxic flies fed on sucrose diet supplemented with putrescine or spermidine. These results were not surprising. Putrescine and spermidine are located downstream of ODC in the polyamine synthesis pathway (Figure 3) and their actions should be insensitive to DFMO. Taken together these results suggested that polyamine synthesis probably contributed to the lifeshortening effect of dietary amino acids.

\section{Polyamines and HIF-I $\alpha$ /Sima stabilization}

Hypoxia-inducible factor 1 (HIF-1) is a heterodimeric transcription factor that functions as a master regulator of oxygen homeostasis in mammals [12]. The Drosophila homologue of HIF- $1 \alpha$ is similar (sima). It is now well established that an accumulation of HIF- $1 \alpha /$ sima under hypoxic conditions promotes hypoxic tolerance [13]. Recent studies have suggested a possible mechanism that may link polyamines and HIF-1 $\alpha$ dependent hypoxic adaptation. Spermidine/spermine acetyl transferase is the rate limiting enzyme for polyamine retroconversion (Figure 3). It is induced by spermidine and spermine [14]. It associates to HIF- $1 \alpha$ and reduces its activity by promoting its ubiquitination and its degradation by the proteasone $[15,16]$. We therefore asked whether HIF- $1 \alpha /$ sima contributed to the actions of dietary amino acids and polyamines on chronically hypoxic flies.

HIF- $1 \alpha /$ sima signalling in response to chronic hypoxia was evaluated using the endogenous lactate dehydrogenase activity of adult $w^{1118}$ flies. We also used adult LDHLacZ reporter flies in which expression of $\beta$-galactosidase was placed under the control of a strong hypoxia sensitive, murine LDH-A enhancer [17]. We observed that chronic hypoxic conditions $\left(5 \% \mathrm{O}_{2}, 24\right.$ or 48 hours) did not increase activities of $\beta$-galactosidase or of endogenous lactate dehydrogenase. Anoxic conditions $\left(<0.1 \% \mathrm{O}_{2}, 1-2\right.$ hours) increased activities of the reporter proteins about 2 fold. These results indicated that hypoxic signalling in adult flies only responded to strong anoxic stresses.

We then used sima loss of function mutants (sima ${ }^{07607 /}$ 07607). These flies develop normally under normoxic conditions. They did not under hypoxic conditions, meaning a decreased hypoxic tolerance [13]. Sima ${ }^{07607 / 07607}$ flies were raised under normoxic conditions and newly emerging adults were exposed to chronic hypoxic conditions. Figure 6 shows that dietary casein decreased the longevity of hypoxic sima ${ }^{07607 / 07607}$ flies fed on a $10 \%$ sucrose diet. Thus, the life-shortening effect of casein did not require an active sima.

\section{GC7 prevented amino acid and polyamine toxicities}

A well known function of polyamine synthesis is to provide spermidine for the hypusination of eukaryotic initiation factor 5A (eIF5A) [18]. Hypusine ( $\mathrm{N}^{\varepsilon_{-}}$(4-amino-2hydroxybutyl)lysine) is a unique amino acid that occurs only in eiF5A, both in mammals [18] and Drosophila [19]. It is formed by a two step mechanism (Figure 3). First, deoxyhypusine synthase (DHS) transfers the 4-aminobutyl moiety of spermidine to the $\varepsilon$-amino group of a specific lysine residue of inactive eIF5A. Deoxyhypusine hydroxylase then hydroxylates eIF5A-deoxyhypusine into eIF5A-hypusine which is the active form of eIF5A. Potent inhibitors of DHS have been developed and are now established tools to assess the functional role of eIF5A hypusination. The most potent inhibitor known so far is GC7, a guanylated derivative of 1,7-diaminoheptane (DAH) [20].

We asked whether GC7 modified the responses of hypoxic flies to amino acids and polyamines. Figure 7A shows that GC7 $(10 \mathrm{mM})$ did not modify the longevity of hypoxic, starving flies. Figure 7B shows that GC7 decreased the longevity of hypoxic flies which were fed on a $10 \%$ sucrose diet. Figure 7(C-J) further shows that GC7 increased the median and maximum longevities of hypoxic flies fed on casein, L-arginine, L-glutamine, $\mathrm{L}$-asparagine, L-ornithine, L-citrulline, putrescine and spermidine. Percentage changes in mean longevity by GC7 were 28 to $168 \%$. We observed however that flies exposed to GC7 were shorter lived than hypoxic flies fed on a pure sucrose diet (14.2 days). Thus, GC7 partially reversed the life-shortening effects of casein, amino acids and polyamines.

We then used DAH which is chemically related to GC7 and is a 500 times weaker inhibitor of DHS [20]. DAH did not reduce the life-shortening effects of L-arginine (Figure 7D) or of L-citrulline (Figure 7H). These results suggested that GC7 protected hypoxic flies against amino acid toxicity by a DHS dependent mechanism. 
A Phosphate buffered saline

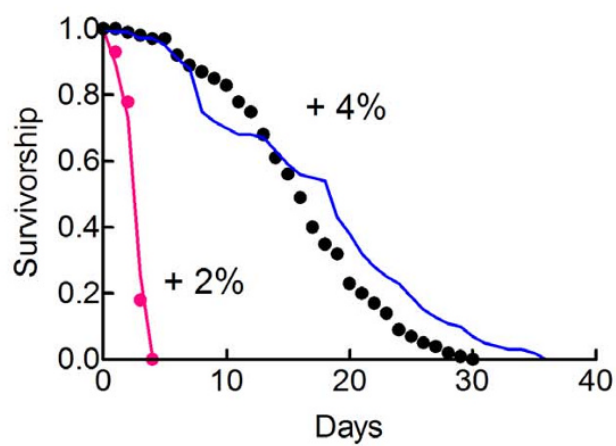

C
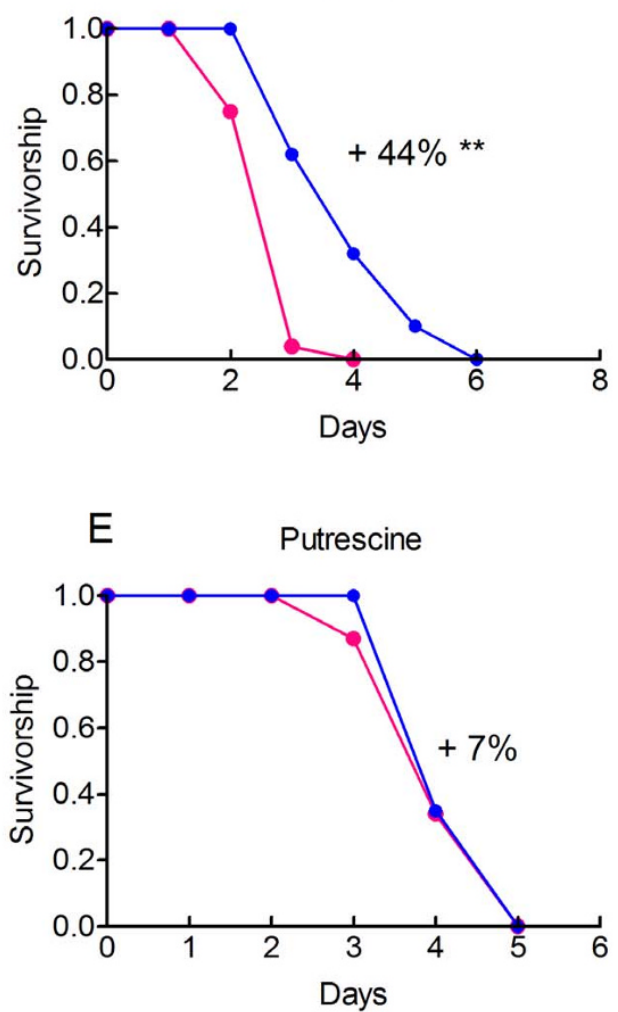

B

Casein

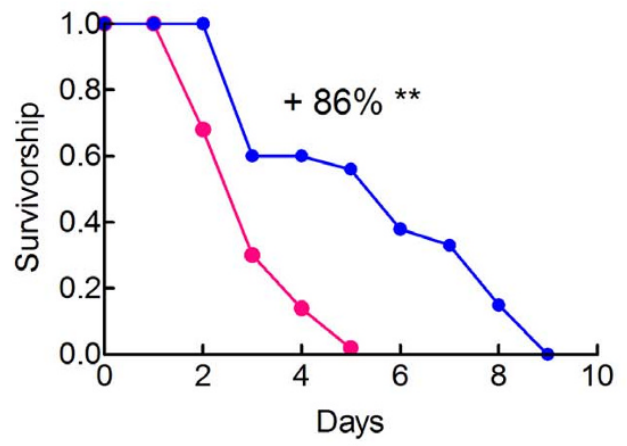

D

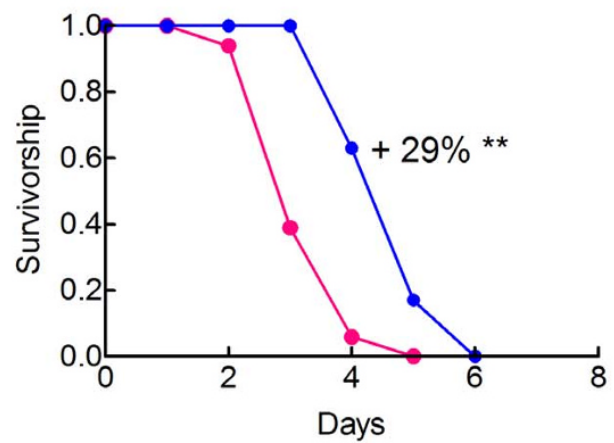

F

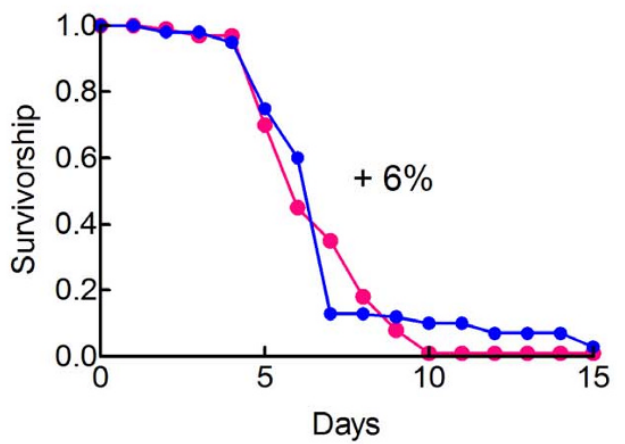

\section{Figure 5}

DFMO prevents actions of amino acid but not of polyamines. Hypoxic flies were fed on a $10 \%$ sucrose diet supplemented with $10 \mathrm{mM}$ of the indicated compounds and survivorship curves were determined. Experiments were performed in the absence or the presence of 10 mM DFMO. Percentage changes in longevity by DFMO are indicated for each dietary condition. A log rank test was used to compare survivorship curves. $* *$ : $p<0.000 \mathrm{I}$, no asterisk: $\mathrm{p}>0.00 \mathrm{I}$. A. Control conditions. Curves on the left are survivorship curves obtained under starvation conditions in the absence (dots, $n=50)$ or the presence (curve, $n=80$ ) of $10 \mathrm{mM}$ DFMO. Curves on the right are survivorship curves obtained with a $10 \%$ sucrose medium in the absence (dots, $n=237$ ) or the presence (curve, $n=118$ ) of $10 \mathrm{mM}$ DFMO. B-F. $10 \%$ sucrose diet supplemented with $0.1 \%$ casein or $10 \mathrm{mM}$ amino acid or polyamine as indicated. Experiments were performed in the absence (magenta) or the presence of 10 mM DFMO (blue). Sample sizes were B (control, I89; DFMO, 48), C (control, I30; DFMO, 88), D (control, 208; DFMO, 59), E (control, I46; DFMO, 48) and F (control, I I0; DFMO, 60). 


\section{The hypoxic sensitivity of flies of different strains}

The responses of Drosophila to changes in diet are well known to be highly dependent on genetic background [21]. It was important to determine that the conclusions obtained with the $w^{1118}$ flies were strain independent. Figure 8 shows that the longevities of hypoxic Canton $S$ and Oregon $\mathrm{R}$ flies were shortened by casein and by L-ornithine. In addition, GC7 relieved the life-threatening effect of L-ornithine in the two strains of flies.

\section{Discussion}

Chronic hypoxic conditions unmask an unexpected lifeshortening effect of dietary proteins and amino acids $[6,8]$. This paper uses pharmacological tools to analyse the mechanisms involved. First we observed that any one of the amino acids which are incorporated into proteins decreased the longevity of hypoxic flies fed on a sucrose diet. Their half maximum actions are observed at low $\mathrm{mM}$ concentrations. The concentrations of amino acids provided by standard food media such as the one used to maintain our stocks of flies are 100 times larger $(1.7 \%$ yeast, $\sim 80 \mathrm{mM}$ ). Thus, low "physiological" concentrations of amino acids such as the one experienced under natural conditions decrease the longevity of hypoxic flies. It is important to stress that a life-shortening effect of dietary amino acids is only observed under chronic hypoxic conditions. Dietary amino acids are required for the normal

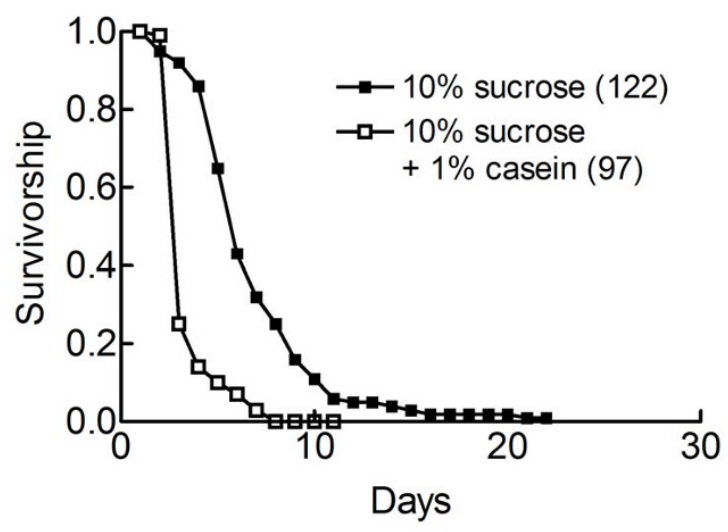

Figure 6

Casein decreases the longevity of sima loss of function mutants. Sima $07607 / 07607$ flies were raised under normoxic conditions. Newly emerged adults were shifted to chronic hypoxic conditions and maintained either on a pure $10 \%$ sucrose diet or a $10 \%$ sucrose diet supplemented with $1 \%$ casein and survivorship curves were determined. Sample sizes are indicated in parentheses. Survivorship curves were compared using the log-rank test. $P$ was $<0.000 \mathrm{I}$. Corresponding survivorship curves for $w^{1118}$ flies are presented in Figure IC. development of the flies and they increase the longevity of normoxic adult flies [6].

Polyamine metabolism is an evolutionary conserved metabolic pathway. In mammals, polyamines are essential for normal cell growth and differentiation [22,23]. Pharmacological depletion of tissue polyamines and the inhibition of eIF5A hypusination are usually cytostatic and antiapoptotic [24-27]. Polyamine metabolism in insects is less documented. All enzymes of the polyamine synthetic pathway are encoded by the Drosophila genome. Spermidine is major form of polyamines in developing Drosophila [28] and transcripts for ODC are expressed in tissues from adult flies [29]. Gutfeeling, is the Drosophila homolog of antizyme, an endogenous inhibitor of ODC. Mutations in gutfeeling cause defects in embryonic peripheral and central nervous systems [30]. Treatment of adult crickets (Acheta domesticus) with DFMO decreases spermidine levels and this decrease can be overcome by exogenous putrescine [11]. In the same species, putrescine has a mitogenic effect on mushroom body neuronal precursors. Spermidine and spermine do not induce neuroblast proliferation but they induce neurite outgrowth [31]. Taken together, these studies indicate that polyamines are required for the normal development of insects. We further show here that adult normoxic flies tolerate large amounts of polyamine in their food. This is not surprising for wild flies live on rotten fruits which are full of polyamines. The opposite effect is observed under chronic hypoxic conditions. Putrescine, spermidine and spermine decrease the longevity of chronically hypoxic flies.

The pharmacological evidence presented here suggests that the synthesis of polyamines contribute to the life shortening effect of amino acids.

(i) Actions of yeast extracts, casein and individual amino acids are reproduced by L-ornithine, L-citrulline, two amino acid intermediates of the urea cycle and by polyamines (putrescine, spermine and spermidine). Lornithine and L-citrulline are as potent as natural amino acids. Polyamines are less potent than amino acids. The difference probably results from pharmacokinetic reasons. Amino acids and polyamines enter cells via different transporters [32]. They may be metabolised to different extents before reaching their targets.

(ii) Inhibition of ODC with DFMO prevents the toxicity of a casein hydrolysate and of all individual amino acids that have been tested. DFMO does not prevent actions of putrescine or of spermidine which are located downstream of ODC in the polyamine synthetic pathway (Figure 3). 

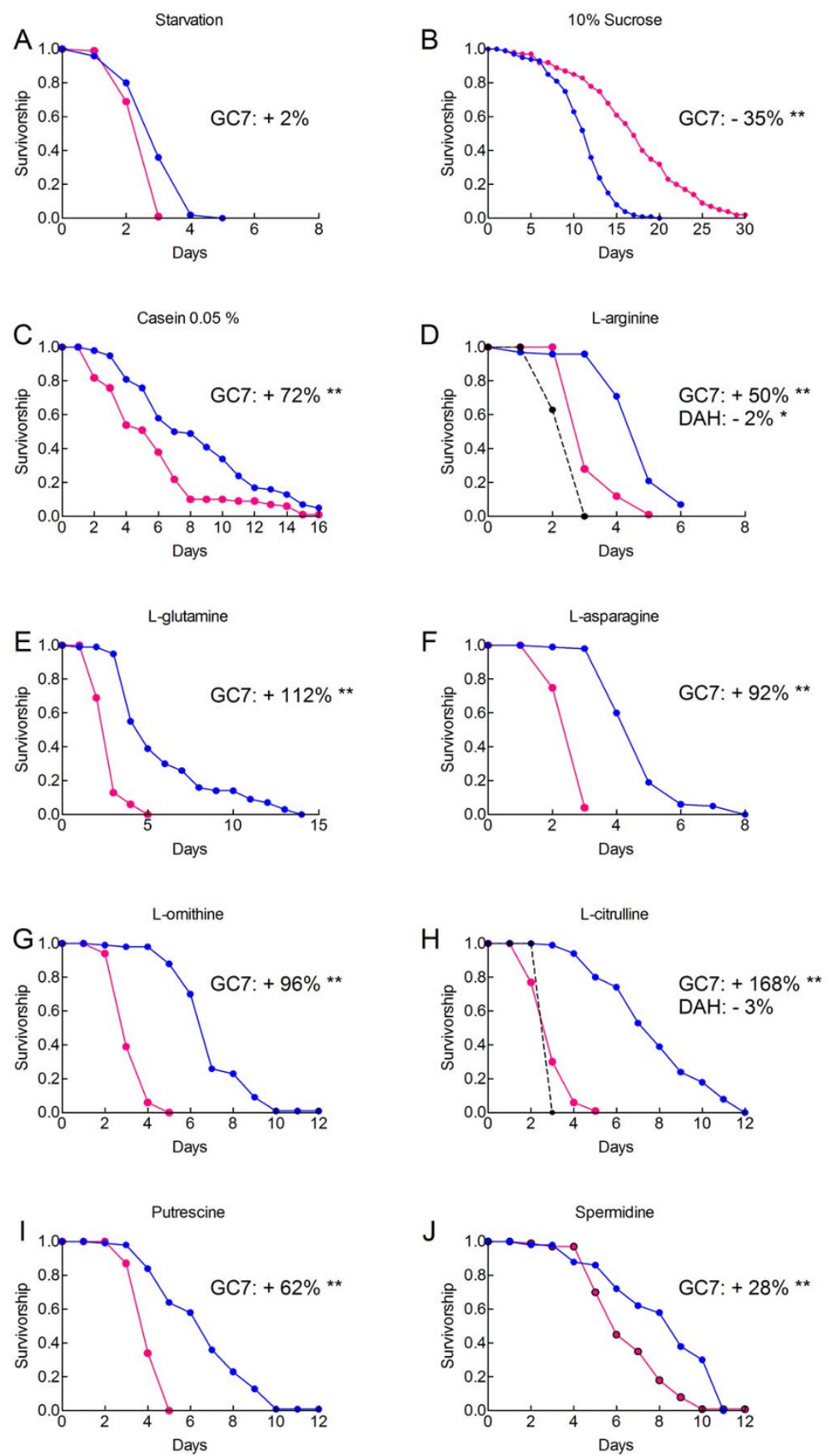

\section{Figure 7}

GC7 prevents actions of casein, amino acids and polyamines. Survivorship curves were determined in the absence (magenta) or the presence of $10 \mathrm{mM} \mathrm{GC7}$ (blue) or of $10 \mathrm{mM} \mathrm{DAH}$ (dotted line in panels D and H). Percentage changes in longevity by GC7 or DAH are indicated for each dietary condition. A log rank test was used to compare survivorship curves. **: $\mathrm{p}<0.000 \mathrm{I}$, *: $\mathrm{p}<0.00 \mathrm{I}$, no asterisk: $\mathrm{p}>0.00 \mathrm{I}$. Dietary conditions were: A, starvation, B, $10 \%$ sucrose diet, $\mathrm{C}, 10 \%$ sucrose diet supplemented with $0.05 \%$ casein, D-F, $10 \%$ sucrose diet supplemented with $10 \mathrm{mM}$ amino acids or polyamines as indicated. Sample sizes were: A (50 each), B (control, 237; GC7, 99), C (control, 79; GC7, 86), D (control, I79; GC7, I35; DAH, 40), E (control I08; GC7, I03) and F (control, I30; GC7, I93), G (control, 208; GC7, 80), H (control, 208; GC7, 80), I (control, I62; GC7, 89; DAH, 40) and J (control, I I0; GC7, 50). 

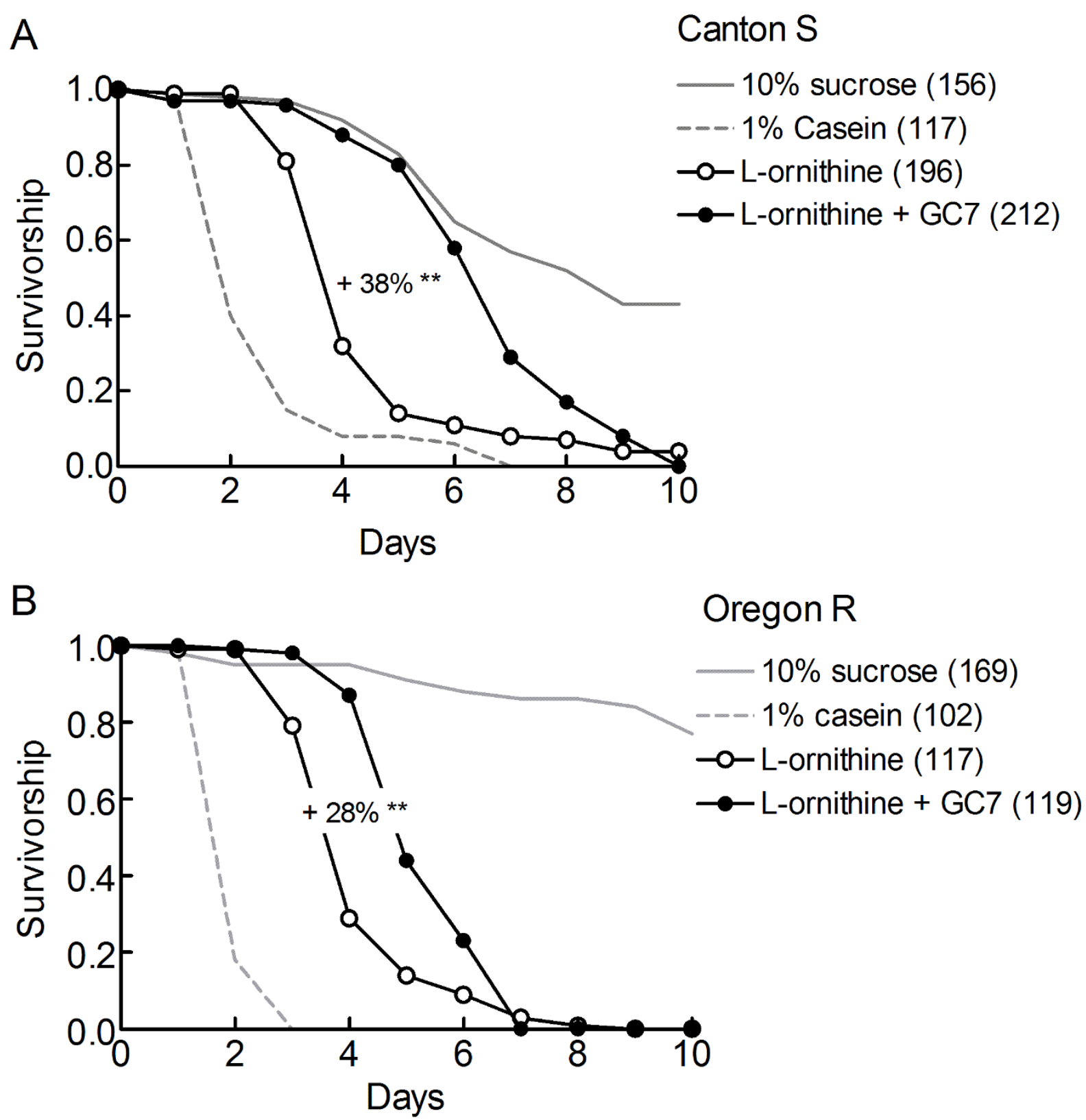

Figure 8

The longevities of hypoxic Canton $\mathbf{S}$ and Oregon $\mathbf{R}$ flies are diet and GC7 sensitive. Flies were fed on different diets as indicated and survivorship curves were defined under chronic hypoxic conditions. Sample sizes are indicated in parentheses. L-ornithine and GC7 were used at $10 \mathrm{mM}$. Percentage changes in longevity by GC7 are indicated. A log rank test was used to compare survivorship curves: $* *$ : $p<0.000$ I. The survivorship curves of flies fed on a $10 \%$ sucrose medium were truncated for clarity. Median and maximum longevities of hypoxic flies fed on a 10\% sucrose diet were: Canton S, 8 days and 22 days, Oregon $\mathrm{R}, \mathrm{I} 4$ days and 22 days. 
Polyamines have several molecular targets and they often act by more than one type of mechanism [22-27]. (i) They interact with nucleic acids and regulate the transcription of a number of genes including the antizyme, an endogenous ODC inhibitor [33] and spermidine/spermine $\mathrm{N}^{1}$ acetyltransferase, the rate limiting enzyme for polyamine retroconversion [14]. (ii) Polyamines interact with phospholipids and negatively charged protein domains. They influence the activity of ion channels such as NMDA receptors, AMPA receptors, $\mathrm{K}^{+}$channels and $\mathrm{Ca}^{2+}$ channels [22]. They activate kinases such as casein kinase and Cdc7 [34]. (iii) Finally, spermidine is a precursor for the hypusination of eIF5A (Figure 3 ). We show here that the toxicities of amino acids and polyamines are partially prevented by GC7, a specific inhibitor of DHS. A chemically related drug, DAH, is inactive. These suggest that DHS contributes to the life-shortening effect of amino acids, possibly by promoting hypusination of eIF5A. The contribution of other targets of polyamines cannot be excluded.

We also observed DFMO and GC7 are unable to fully prevent the toxicities of amino acids and polyamines. Different reasons may account for their partial actions.

(i) DFMO and GC7 act as competitive antagonists of ODC and DHS respectively $[10,20]$. As a consequence, their potency in vivo can be limited by the presence of large concentrations of enzyme substrates (L-ornithine and spermidine).

(ii) GC7 by itself reduces the survival of sucrose fed hypoxic flies. This effect may limit its beneficial action when flies are fed on amino acids.

(iii) It is well known that polyamine metabolism is highly complex in mammals and that perturbations of polyamine synthesis induce compensatory changes that eventually reduce the efficacy of the drugs used $[33,35,36]$.

(iv) Finally, the possibility that polyamines reduce the longevity of chronically hypoxic flies by more than one mechanism cannot be excluded.

A well known action of polyamines in mammals is to stimulate protein synthesis [37]. A simple hypothesis could be that high rates of protein synthesis are toxic to energetically compromised hypoxic tissues. Accordingly reducing translation could promote survival by sparing energy. This hypothesis is unlikely.

(i) Single amino acid species produce the same effect as a casein hydrolysate; they are unable to support protein synthesis. (ii) Inhibiting translation with cycloheximide does not reproduce the actions of DFMO or of GC7 (data not shown).

(iii) Although eIF5A has initially been described as a general translation factor [38], more recent evidence suggests that hypusination of eIF5A does not regulate the overall rates of protein synthesis both in Drosophila [39] and mammalian cells [40]. Other functions of eIF5A have been proposed. They are nuclear export and RNA turnover [41,42], p53 dependent apoptosis [43] and neuronal survival [44]. Further work is required to define the possible contributions of these different mechanisms to amino acid dependent hypoxic tolerance.

Results presented here identify a link between the synthesis of polyamines and the longevity of chronically hypoxic Drosophila. Circumstantial evidence suggests that a similar link probably exist in mammals. For instance it has been reported that activity of ODC and tissue polyamines levels increase in different models of cardiac and brain ischemia [45-47]. In addition, DFMO has neuroprotective $[46,48]$ and cardioprotective [49] actions in mammals. The molecular mechanisms involved have not been analyzed. The possibility that inhibition of polyamine synthesis protects ischemic mammalian tissues by preventing eIF5A hypusination has not yet been considered.

\section{Conclusion}

Chronic hypoxic conditions unmask a life-shortening effect of dietary amino acids. Pharmacological evidence obtained so far suggests that the synthesis of polyamines and the hypusination of eIF5A contribute to the life-shortening effect of dietary amino acids. The results also indicate that drugs can be designed to specifically enhance the hypoxic tolerance of an organism.

\section{Methods \\ Fly Strains}

$w^{1118}$ flies were used in all experiments unless otherwise indicated. This strain is long lived under normoxic conditions. The mean longevity of $\mathrm{w}^{1118}$ flies maintained under optimum diet conditions (10\% sucrose and 5\% yeast) was $78.6 \pm 2.2$ days $(n=88)$ [6]. Oregon $R$ and Canton $S$ flies were used in some experiments. All flies were obtained from the Bloomington stock center. Sima ${ }^{07607 /}$ TM3 flies and LDH-LacZ reporter flies were kindly provided by Dr P. Wappner. Homozygous, loss of function sima $07607 / 07607$ flies, were generated by crossing heterozygous sima ${ }^{07607 / T M 3}$ flies. Sima ${ }^{07607 / T M 3}$ flies were identified to their stubble phenotype.

All flies were reared in $300 \mathrm{ml}$ bottles filled with $30 \mathrm{ml}$ of standard food medium (8.2\% cornmeal, $6.2 \%$ sucrose, $1.7 \%$ heat inactivated baker's yeast and $1 \%$ agar supple- 
mented with $3.75 \mathrm{~g} / \mathrm{l}$ methyl 4-hydroxybenzoate). They were maintained in humidified, temperature controlled chambers at $25^{\circ} \mathrm{C}, 60 \%$ relative humidity and under a 12:12 light: dark cycle.

\section{Chemicals}

N1-Guanyl-1,7-diaminoheptane (GC7) was from Research Technologies Inc. (Novato, Ca). All other chemical were purchased from the Sigma Chemical Co (St Louis, Mo).

\section{Survival under chronic hypoxic conditions}

All experiments were performed using males. Newly emerging males were collected over a $24 \mathrm{~h}$ period and divided into identical batches of 10 males per vial. Vials were sealed with cotton plugs (normoxic conditions) or with natural rubber septa (SubA seal, Sigma, St Louis, Mo) for hypoxic/anoxic conditions. The atmosphere was changed to a $5 \% \mathrm{O}_{2}$ (hypoxia) or a $0.1 \% \mathrm{O}_{2}$ (anoxia) atmosphere by flushing vials with premixed gas $\mathrm{O}_{2} / \mathrm{N}_{2}$ mixtures (Linde Gas) [8]. Dead flies were counted every day. Flies were maintained at $25^{\circ} \mathrm{C}$ and a $12: 12$ light: dark cycle.

\section{Dietary manipulations}

Solutions of sucrose, heat inactivated yeast, casein hydrolysate (referred to as casein), amino acids, polyamines and inhibitors were prepared at room temperature in phosphate buffered saline. All solutions were prepared immediately before use and were $\mathrm{pH}$ checked (7.5). Two $\mathrm{ml}$ aliquots were used to wet pieces of Kleenex ${ }^{\circledR}$ towels (1/4 of the original size) that had previously been inserted into the tubes. Nutrient media were labelled according to their sucrose and yeast contents. A $10 \mathrm{~S} 10 \mathrm{Y}$ nutrient medium contained $10 \%$ sucrose $(\mathrm{S})$ and $10 \%$ yeast $(\mathrm{Y})$. In starvation experiments (OSOY nutrient medium), flies were exposed to pieces of Kleenex ${ }^{\circledR}$ towels wetted with $2 \mathrm{ml}$ of phosphate buffered saline to prevent dehydration.

\section{Toxicity assay}

Solutions of drugs $(10 \mathrm{mM})$ in phosphate buffered saline supplemented with $10 \%$ sucrose were prepared and used to wet pieces of Kleenex ${ }^{\circledR}$ tissue. Ten, one day old, male flies were added to each tube and tubes were sealed with cotton plugs. Dead flies were counted after 10 days. The 10 day mortality rate was $2 \%$ in the absence of drugs. It was $<5 \%$ in the presence of amino acids, polyamines or drugs.

\section{Beta-galactosidase assay}

Frozen LDH-LacZ reporter flies were homogenized into lysis buffer (50 mM Hepes, 5 mM CHAPS at pH 7.5) at $4{ }^{\circ} \mathrm{C}$. Extracts were centrifuged for 30 minutes at 13,200 $\mathrm{rpm}$. The supernatant was recovered. Beta-galactosidase activity was measured on $100 \mu \mathrm{g}$ of protein using the betagalactosidase reporter gene activity kit (Sigma Chemical Co. St Louis, Mo). The time of incubation at $37^{\circ} \mathrm{C}$ was 1 hour.

\section{Lactate dehydrogenase activity}

Frozen $w^{1118}$ flies were homogenized in phosphate buffered saline ( $\mathrm{pH} 7.5$ ) supplemented with $0.12 \mathrm{M}$ mannitol, $0.22 \mathrm{M}$ sucrose and $1 \mathrm{mM}$ EDTA at $4^{\circ} \mathrm{C}$. Extracts were centrifuged for 30 minutes at 13,200 rpm. The supernatant was recovered. Lactate dehydrogenase activity was measured spectrophotometrically at $25^{\circ} \mathrm{C}$ and using $200 \mu \mathrm{g}$ of protein. The incubation medium was phosphate buffered saline ( $\mathrm{pH} 7.5$ ) supplemented with $30 \mu \mathrm{M}$ pyruvate and $15 \mu \mathrm{M}$ NADH.

\section{Protein assays}

Proteins were measured using the Bradford reagent (Biorad Res. Labs, Hercules, Ca).

\section{Statistical analysis}

Complete survivorship curves were defined for all conditions considered. Survivorship curves were compared using the logrank test and the GraphPad Prism 4 software (San Diego, Ca). For each condition we calculated the mean, median and maximum longevities. The maximum longevity was defined as the median longevity of the final surviving 10\%. Dose response curves for the actions of yeast and casein on the mean survival of hypoxic flies were fitted to sigmoidal curves using the GraphPad Prism 4 software.

\section{Authors' contributions}

PV and CF designed and performed the experiments. CF wrote the paper.

\section{References}

I. Teleman AA, Hietakangas V, Sayadian AC, Cohen SM: Nutritional control of protein biosynthetic capacity by insulin via Myc in Drosophila. Cell Metabolism 2008, 7:21-32.

2. Hinnebusch A: Translational regulation of GCN4 and the general amino acid control of yeast. Ann Rev Microbiol 2005, 59:407-450.

3. Bordone L, Guarente L: Calorie restriction, SIRT I and metabolism: understanding longevity. Nature Rev Mol Cell Biol 2005, 6:298-304.

4. Min K-J, Tatar M: Restriction of amino acids extends lifespan in Drosophila melanogaster. Mech Ageing Dev 2006, I 27:643-646.

5. Bass TM, Grandisson RC, Wong R, Martinez P, Partridge L, Piper MDW: Optimization of dietary restriction protocols in Drosophila. J Gerontol A Biol Sci Med Sci 2007, 62: I07|-I08I.

6. Vigne $P$, Frelin C: Diet dependent longevity and hypoxic tolerance of adult Drosophila melanogaster. Mech Ageing Dev 2007, I 28:40 I-406.

7. Haddad GG: Tolerance to low $\mathbf{O}_{2}$ : lessons from invertebrate genetic models. Exp Physiol 2006, 91 :277-282.

8. Vigne P, Frelin C: A low protein diet increases the hypoxic tolerance in Drosophila. PLoS ONE 2006, I:e56.

9. Libert S, Zwiener J, Chu X, Vanvoorhies W, Roman G, Pletcher SD: Regulation of Drosophila life span by olfaction and foodderived odors. Science 2007, 3 15: I|33-1 | 37. 
10. Mamont PS, Böhlen P, McCann PP, Bey P, Schuber F, Tardif A: Alphamethyl ornithine, a potent competitive inhibitor of ornithine decarboxylase, blocks proliferation of rat hepatoma cells in culture. Proc Natl Acad Sci USA 1976, 73:1626-1630.

II. Cayre M, Strambi C, Charpin P, Augier R, Strambi A: Specific requirement of putrescine for the mitogenic action of juvenile hormone on adult insect neuroblasts. Proc Natl Acad Sci USA 1997, 94:8238-8242.

12. Semenza GL: HIF-I, O(2), and the 3 PHDs: how animal cells signal hypoxia to the nucleus. Cell 200I, 107: I-3.

13. Centanin L, Ratcliffe PJ, Wappner P: Reversion of lethality and growth defects in Fatiga oxygen-sensor mutant flies by loss of hypoxia-inducible factor alpha/Sima. EMBO Rep 2005, 6:1070-1075.

14. Butcher NJ, Broadhurst GM, Minchin RF: Polyamine-dependent regulation of spermidine-spermine $\mathrm{NI}$-acetyltransferase mRNA translation. I Biol Chem 2007, 282:28530-28539.

15. Baek JH, Liu YV, McDonald KR, Wesley JB, Hubbi ME, Byun H, Semenza GL: Spermidine/spermine N(I)-acetyltransferase-I binds to hypoxia-inducible factor-Ialpha (HIF-Ialpha) and RACKI and promotes ubiquitination and degradation of HIF-I alpha. J Biol Chem 2007, 282:23572-23580.

16. Baek JH, Liu YV, McDonald KR, Wesley JB, Zhang H, Semenza GL: Spermidine/spermine-N I-acetyltransferase 2 is an essential component of the ubiquitin ligase complex that regulates hypoxia-inducible factor Ialpha. I Biol Chem 2007, 282:33358-33366.

17. Lavista-Llanos S, Centanin L, Irisarri M, Russo DM, Gleadle JM, Bocca SN, Muzzopappa M, Ratcliffe PJ, Wappner P: Control of the hypoxic response in Drosophila melanogaster by the basic helix-loop-helix PAS protein similar. Mol Cell Biol 2002, 22:6842-6853.

18. Park $\mathrm{MH}$ : The post-translational synthesis of a polyaminederived amino acid, hypusine, in the eukaryotic translation initiation factor 5A (elF5A. J Biochem 2006, I39: I6I- I69.

19. Gordon ED, Mora R, Meredith SC, Lee C, Lindquist SL: Eukaryotic initiation factor 4D, the hypusine-containing protein, is conserved among eukaryotes. J Biol Chem 1987, 262:I6585-I6589.

20. Jakus J, Wolff EC, Park MH, Folk JE: Features of the spermidinebinding site of deoxyhypusine synthase as derived from inhibition studies. Effective inhibition by bis- and mono-guanylated diamines and polyamines. I Biol Chem 1993, 268: $1315 \mid-13159$.

21. Pletcher SD, Libert S, Skorupa D: Flies and their golden apples: the effect of dietary restriction on Drosophila aging and agedependent gene expression. Aging Res Rev 2005, 4:45I-480.

22. Wallace HM, Fraser AV, Hughes A: A perspective of polyamine metabolism. Biochem / 2003, 376: I- |4.

23. Gerner EW, Meyskens F: Polyamines and cancer: old molecules, new understanding. Nature Rev Cancer 2004, 4:781-792.

24. Stefanelli C, Stanic I, Zini M, Bonavita F, Flamigni F, Zambonin L, Li L, Pignatti C, Guarnieri C, Caldarera CM: Polyamines directly induce release of cytochrome $c$ from heart mitochondria. Biochem J 2000, 347:875-880.

25. Thomas T, Thomas T): Polyamines in cell growth and cell death: molecular mechanisms and therapeutic applications. Cell $\mathrm{Mol}$ Life Sci 200I, 58:244-258.

26. Lee Y, Kim HK, Park HE, Park MH, Joe YA: Effect of N I-guanylI,7-diaminoheptane, an inhibitor of deoxyhypusine synthase, on endothelial cell growth, differentiation and apoptosis. Mol Cell Biochem 2002, 237:69-76.

27. Wallace HM, Fraser AV: Inhibitors of polyamine metabolism. Amino Acids 2004, 26:353-365.

28. Callaerts $P$, Geuns ], De Loof A: Polyamine changes during early development of Drosophila melanogaster. J Insect Physiol 1992, 38:75I-758.

29. Rom E, Kahana C: Isolation and characterization of the Drosophila ornithine decarboxylase locus: evidence for the presence of two transcribed ODC genes in the Drosophila genome. DNA Cell Biol 1993, I 2:499-508.

30. Salzberg A, Golden K, Bodmer R, Bellen HJ: gutfeeling, a Drosophila gene encoding an antizyme-like protein, is required for late differentiation of neurons and muscles. Genetics 1996, 144:183-196.

31. Cayre M, Malaterre J, Strambi C, Charpin P, Ternaux JP, Strambi A: Short- and long-chain natural polyamines play specific roles in adult cricket neuroblast proliferation and neuron differentiation in vitro. J Neurobiol 2001, 48:315-324.

32. Romero-Calderón $R$, Krantz DE: Transport of polyamines in Drosophila S2 cells: kinetics, pharmacology and dependence on the plasma membrane proton gradient. Biochem J 2006, 393:583-589.

33. Coffino P: Regulation of cellular polyamines by antizyme. Nature Rev Mol Cell Biol 200I, 2: 188-194.

34. Kakusho N, Taniyama C, Masai H: Identification of stimulators and inhibitors of CDC7 kinase in vitro. J Biol Chem 2008, 283:1921।-19218.

35. Nishimura K, Murozumi K, Shirahata A, Park MH, Kashiwagi K, Igarashi K: Independent roles of elF5A and polyamines in cell proliferation. Biochem J 2005, 385:779-785.

36. Wallace $\mathrm{H}$, Niiranen K: Polyamine analogues - an update. Amino Acids 2007, 33:26I-265.

37. Yoshida M, Meksuriyen D, Kashiwagi K, Kawai G, Igarashi K: Polyamine stimulation of the synthesis of oligopeptide-binding protein (OppA). Involvement of a structural change of the Shine-Dalgarno sequence and the initiation codon aug in oppa mRNA. J Biol Chem 1999, 274:22723-22728.

38. Kemper WM, Berry KW, Merrick WC: Purification and properties of rabbit reticulocyte protein synthesis initiation factors M2Balpha and M2Bbeta. J Biol Chem 1976, 25 I:555 I-5557.

39. Gordon ED, Mora R, Meredith SC, Lindquist SL: Hypusine formation in eukaryotic initiation factor $4 D$ is not reversed when rates or specificity of protein synthesis is altered. $\mathrm{J} \mathrm{Biol} \mathrm{Chem}$ 1987, 262:16590-16595.

40. Zanelli CF, Valentini SR: Is there a role for elF5A in translation. Amino Acids 2007, 33:35 I-358.

4I. Ruhl M, Himmelspach M, Bahr GM, Hammerschmid F, Jaksche $H$ Wolff B, Aschauer H, Farrington GK, Probst H, Bevec D, Hauber J: Eukaryotic initiation factor $\mathbf{5 A}$ is a cellular target of the human immunodeficiency virus type I Rev activation domain mediating trans-activation. I Cell Biol 1993, I 23:1309-1320.

42. Hauber I, Bevec D, Heukeshoven J, Krätzer F, Horn F, Choidas A Harrer T, Hauber J: Identification of cellular deoxyhypusine synthase as a novel target for antiretroviral therapy. J Clin Invest 2005, I I 5:76-85.

43. Li AL, Li HY, Jin BF, Ye Q, Zhou T, Yu XD, Pa X, Man JH, He K, Yu $M$, Hu MR, Wang J, Yang SC, Shen BF, Zhang XM: A novel elF5A complex functions as a regulator of p53 and p53-dependent apoptosis. J Biol Chem 2004, 279:4925I-49258.

44. Huang $Y$, Higginson DS, Hester L, Park MH, Snyder SH: Neuronal growth and survival mediated by eIF5A, a polyamine-modified translation initiation factor. Proc Natl Acad Sci USA 2007. 104:4194-4199.

45. Longo LD, Packianathan S, McQueary JA, Stagg RB, Byus CV, Cain $C D$ : Acute hypoxia increases ornithine decarboxylase activity and polyamine concentrations in fetal rat brain. Proc Nat Acad Sci USA 1003, 90:692-696.

46. Babu GN, Sailor KA, Beck J, Sun D, Dempsey RJ: Ornithine decarboxylase activity in in vivo and in vitro models of cerebral ischemia. Neurochem Res 2003, 28:1851-1857.

47. Zhao YJ, Xu CQ, Zhang WH, Zhang L, Bian SL, Huang O, Sun HL, Li QF, Zhang YQ, Tian Y, Wang R, Yang BF, Li WM: Role of polyamines in myocardial ischemia/reperfusion injury and their interactions with nitric oxide. Eur J Pharmacol 2007, 562:236-246.

48. Pernet $\mathrm{V}$, Bourgeois $\mathrm{P}, \mathrm{Di}$ Polo A: A role for polyamines in retinal ganglion cell excitotoxic death. I Neurochem 2007, |03:|48|-|490.

49. Tantini B, Fiumana E, Cetrullo S, Pignatti C, Bonavita F, Shantz LM, Giordano E, Muscari C, Flamigni F, Guarnieri C, Stefanelli C, Caldarera CM: Involvement of polyamines in apoptosis of cardiac myoblasts in a model of simulated ischemia. I Mol Cell Cardio 2006, 40:775-782. 